\title{
Inoculating Against Fake News About COVID-19
}

\author{
Sander van der Linden ${ }^{1 *}$, Jon Roozenbeek ${ }^{1}$ and Josh Compton ${ }^{2}$ \\ ${ }^{1}$ Cambridge Social Decision-Making Laboratory, Department of Psychology, School of Biology, University of Cambridge, \\ Cambridge, United Kingdom, ${ }^{2}$ Institute for Writing and Rhetoric, Dartmouth College, Hanover, NH, United States
}

The outbreak of the SARS-CoV-2 novel coronavirus (COVID-19) has been accompanied by a large amount of misleading and false information about the virus, especially on social media. In this article, we explore the coronavirus "infodemic" and how behavioral scientists may seek to address this problem. We detail the scope of the problem and discuss the negative influence that COVID-19 misinformation can have on the widespread adoption of health protective behaviors in the population. In response, we explore how insights from the behavioral sciences can be leveraged to manage an effective societal response to curb the spread of misinformation about the virus. In particular, we discuss the theory of psychological inoculation (or prebunking) as an

OPEN ACCESS

Edited by:

Gianluca Castelnuovo, Catholic University of the Sacred Heart, Italy

Reviewed by: Kai Shu,

Illinois Institute of Technology,

United States

Raihan Jamil,

Zayed University, United Arab Emirates

*Correspondence:

Sander van der Linden sander.vanderlinden@ psychol.cam.ac.uk

Specialty section: This article was submitted to Health Psychology, a section of the journal Frontiers in Psychology

Received: 09 June 2020 Accepted: 05 October 2020 Published: 23 October 2020

Citation: van der Linden S, Roozenbeek J and Compton J (2020) Inoculating Against Fake News About COVID-19.

Front. Psychol. 11:566790 doi: 10.3389/fpsyg.2020.566790 efficient vehicle for conferring large-scale psychological resistance against fake news.

Keywords: COVID-19, fake news, misinformation, inoculation, infodemic

\section{INTRODUCTION}

The emergence of the novel coronavirus (SARS-CoV-2) in December of 2019 has quickly led to a global pandemic claiming hundreds of thousands of deaths worldwide already (Roser et al., 2020). In the absence of an effective treatment or vaccine, researchers have pointed out that managing the pandemic response will require leveraging insights from the social and behavioral sciences, particularly with regard to non-pharmaceutical interventions and containing the spread of misinformation about COVID-19 (Depoux et al., 2020; Habersaat et al., 2020; Van Bavel et al., 2020). In fact, the spread of misleading information about the virus has led the World Health Organization (WHO) to warn of an on-going "infodemic" or an overabundance of informationespecially misinformation-during an epidemic (World Health Organization, 2020b; Zarocostas, 2020). This makes it harder for people to find trustworthy and reliable information when they need it. In this article, we ask three critical questions to help better inform societal response to the infodemic, namely; (1) what is the scope and reach of misinformation about COVID-19 in the general population, (2) what evidence is there to suggest that misinformation about the virus is undermining public support for-and the adoption of - preventative health behaviors; and (3) how can insights from psychology be leveraged to effectively manage societal response to help limit the spread of influential misinformation? In particular, in order to "immunize" people against the misinformation virus we draw on the theory of psychological inoculation and its real-world application.

\section{MISINFORMATION ABOUT COVID-19}

Misinformation about COVID-19 has proliferated widely on social media, ranging from the peddling of fake "cures," such as gargling with lemon or salt water and injecting yourself with bleach 
(World Health Organization, 2020a), to false conspiracy theories that the virus was bioengineered in a lab in Wuhan (Andersen et al., 2020; Cohen, 2020), or that the 5G cellular network is causing or exacerbating symptoms of COVID-19 (BBC News, 2020). The conspiracy film "Plandemic" appeared online on May 4th of 2020, garnering millions of views and quickly becoming one of the most widespread examples of coronavirusrelated misinformation (Cook et al., 2020). The video promotes dangerous health advice, for example, falsely suggesting that wearing a mask actually "activates" the coronavirus. Fake news about the virus has also been actively promoted by political elites, such as President Trump and Brazilian President Jair Bolsonaro, who falsely claimed that hydroxychloroquine is "working in all places" as a treatment against the virus (Constine, 2020). But misinformation about COVID-19 is not limited to information that is blatantly true or false, which widens the scope of the problem. For example, although the harms and benefits of hydroxychloroquine as a potential treatment are indeed being studied, there is currently no scientific consensus on its effectiveness (Geleris et al., 2020; Meyerowitz et al., 2020). Thus, even deciding what counts as misinformation about COVID19 is a complicated matter, as insights into the causes of and treatments for the virus develop over time. Nonetheless, it is becoming increasingly clear that misinformation about COVID19 is a common problem. For example, a poll by Ofcom in the United Kingdom found that almost half (46\%) of the United Kingdom population reported exposure to fake news about the coronavirus (Ofcom, 2020). Similar results (48\%) have been reported by Pew in the United States (Mitchell and Oliphant, 2020). In particular, amongst those exposed, nearly two-thirds (66\%) reported seeing it on a daily basis, which is problematic as repeated exposure is known to increase belief in fake news (Pennycook et al., 2018). Although mass endorsement of conspiracy theories about the virus is not yet widespread, substantial minorities (typically about a third of the sample) in the United Kingdom and the United States report to believe that the virus is either manmade or produced on purpose by powerful organizations (Freeman et al., 2020; Roozenbeek et al., 2020b; Uscinski et al., 2020). Indeed, a YouGov survey found that about $28 \%$ of Americans and $50 \%$ of Fox News viewers think that Bill Gates is planning to use the COVID19 vaccine to implement microchips in people (Sanders, 2020). Moreover, a recent analysis of the most viewed coronavirus YouTube videos found that over 25\% of the top videos about the virus contained misleading information, reaching over 62 million views worldwide (Li et al., 2020).

\section{HOW IS THE SPREAD OF MISINFORMATION HARMING SOCIETAL RESPONSE TO THE PANDEMIC?}

Another emerging insight is that COVID-19 conspiracies and rampant misinformation can adversely impact the effectiveness of containment strategies. Indeed, misinformation about COVID19 can fundamentally distort people's risk perception of the virus (Krause et al., 2020). This is important as risk perception has been linked to the adoption of COVID-19 preventative health behaviors (Dryhurst et al., 2020). A recent study by Uscinski et al. (2020) found that belief in conspiracies about the virus is associated with a propensity to reject information from expert authorities. Similar findings were reported by Freeman et al. (2020), who also noted a link between belief in COVID-19 conspiracies and an increase in vaccine hesitancy. For example, people who endorsed the conspiracy that the virus is bioengineered were less likely to report compliance with public health guidelines (e.g., staying at home) and were less likely to report to accept a COVID-19 vaccine (see also Imhoff and Lamberty, 2020). These effects are problematic because at present polls show that only $50 \%$ of Americans are willing to get a potential vaccine if one becomes available, which undermines the potential for herd immunity against the coronavirus (Cornwall, 2020). Importantly, misinformation about the virus has been shown to have other serious societal consequences as well. Recent reports have indicated that coronavirus misinformation has been linked to mob attacks, mass poisonings (Depoux et al., 2020), and acts of vandalism (Spring, 2020). In the United Kingdom alone, people have set fire to least 50 phone masts in response to the 5G conspiracy (BBC News, 2020) and research finds that belief in the $5 \mathrm{G}$ conspiracy is linked to violent intentions (Jolley and Paterson, 2020). In addition, an analysis of over 60 million geo-coded cell phones found reduced social distancing in pro-government areas after Brazil's president inaccurately portrayed the risks of COVID-19 (Ajzenman et al., 2020). Similar analyses have been conducted in the United States in response to political polarization over COVID-19 preventative health behaviors (Allcott et al., 2020), highlighting the disruptive potential of high-profile misinformation for both individual and societal well-being.

\section{LEVERAGING INSIGHTS FROM PSYCHOLOGY: INOCULATING AGAINST COVID-19 MISINFORMATION}

So far, little attention has been paid to insights from the social and behavioral sciences to combat misinformation about COVID-19, despite the ample availability of research to draw from Van Bavel et al. (2020). One insight that has emerged is that fact-checks tend to spread slower on social media than misinformation (Vosoughi et al., 2018), making it difficult for fact-checking to be effective on its own. A review by Politico, for example, found that Facebook's fact-checking efforts did little to prevent coronavirus conspiracies from being shared widely in private groups on the platform (Scott, 2020). Further complications arise from the "continued influence effect" of misinformation, which states that people may continue to believe misinformation even after it has been debunked (Ecker et al., 2010; Lewandowsky et al., 2012). In addition, while media literacy initiatives are important and can be effective under the right conditions (Bode and Vraga, 2015; Guess et al., 2020; Van Bavel et al., 2020), they are often expensive to develop, slow to roll out, and reactive rather than proactive.

In particular, given the practical challenges of fact-checking and the difficulty of correcting misinformation after the damage 
is already done, researchers have started to explore prebunking (i.e., preemptive debunking). Because misinformation spreads through networks much like a real virus "infecting its host" and rapidly transmitting falsehoods from one mind to another, the natural antidote is a psychological vaccine against fake news (van der Linden and Roozenbeek, 2020).

\section{Inoculation Theory}

The theory of psychological inoculation takes the historic practice of vaccination in medicine into the realm of resistance to persuasion (McGuire, 1964). In a medical inoculation, a virus is weakened to the point where it will not make the person sick, but it will trigger protective responses, like antibodies. In a persuasion inoculation, a strong challenge (e.g., a conspiracy theory) is weakened to the point where it will not change the person's position-the person's healthy statebut it will trigger protective responses, like enhanced critical thinking (McGuire, 1964; Compton, 2013). In both contexts, a similar process is at work: exposure to weakened challenges leads to resistance to stronger challenges. In psychological inoculation, the weakened challenge often consists of two elements (Compton, 2013), namely; (a) a forewarning of a threat or attack on one's attitudes and (b) a preemptive refutation of counter-arguments (or prebunking). Preemptive refutation of misinformation weakens the misinformation, just as a medical vaccine is often comprised of weakened virus. For example, in a study on misinformation about climate change, participants were (a) forewarned that some political actors try to mislead people on the issue and (b) provided with facts and arguments to refute the misinformation-preemptively-that is, before they were exposed to a full dose of misinformation later on (van der Linden et al., 2017). The study found that the inoculation partially immunized people against climate misinformation (see also Maertens et al., 2020a).

A number of things happen during the inoculation process of resistance to influence. One of the most important is threatthe motivation to engage in resistance. In inoculation research, threat is a response to vulnerability (McGuire, 1964; Compton, 2013) - for example, when a preemptive inoculation message raises and refutes a persuasive attack (e.g., Banas and Richards, 2017), or when an inoculation message exposes reasoning fallacies (Cook et al., 2017). The cognitive and affective processes unleashed by threat are varied and powerful, including increased counterarguing (Pfau et al., 2006), increased attitude accessibility (Pfau et al., 2003), less psychological reactance against the inoculation-informed campaign (Richards and Banas, 2015), and more psychological reactance against attack messages (Miller et al., 2013). For conventional, prophylactic inoculation to take hold, the desired position needs to already be in place-a healthy state (Compton, 2013). This is the classic approach of inoculation theory. In the context of the coronavirus, this would imply protecting the attitudes of those people who are already following public health guidelines. Strengthening their attitudinal defenses will decrease the potency of misinformation attacks. However, a more recent approach within inoculation theory expands its efficacy to also include a therapeutic application-inoculation treatments that target an unhealthy state (Compton, 2020). New work in this latter area expands inoculation theory's reach by inoculating audiences who have already been "afflicted" with the informational virus. Therapeutic inoculation works by boosting immune defenses and decreasing the probability that people will spread the virus. For example, people with skeptical attitudes toward climate science can still benefit from inoculation against misinformation in the sense that they generate stronger attitudes toward the scientific consensus (Cook et al., 2017; van der Linden et al., 2017).

The health domain boasts a particularly strong record for inoculation theory-appropriately enough in the context of COVID-19. Much of this work has looked at how inoculation theory-informed public health messages could help shore up resistance to unhealthy pressures, like smoking cigarettes (Pfau et al., 1992) or binge drinking (Parker et al., 2010). More recently, inoculation work has explored ways of enhancing beneficial health behaviors, like committing to exercise programs (Dimmock et al., 2016) or strengthening vaccination intentions (Wong and Harrison, 2014), especially in response to conspiracy theories (Jolley and Douglas, 2017). For example, vaccination intentions only improved when participants were presented with anti-conspiracy arguments prior to exposure to the vaccination conspiracy theories but not when presented with counter arguments afterward.

\section{Actively Inoculating Against Misinformation}

Two further advances have been proposed in inoculation research that hold promise for the scalability and broad applicability of inoculation interventions, particularly in the context of misinformation: a renewed focus on active inoculations (McGuire and Papageorgis, 1961; Roozenbeek and van der Linden, 2018), and a shift in attention from inoculating against individual examples of unwanted persuasion (e.g., climate change or vaccination) to the manipulation techniques that underpin most fake news such as using emotional language (Brady et al., 2017), conspiratorial reasoning (Lewandowsky et al., 2013; van der Linden, 2015) or impersonating experts online (Goga et al., 2015). The idea behind active inoculation is to let people generate their own "antibodies." A practical application of active inoculation theory is the award-winning online browser game Bad News. ${ }^{1}$ The game offers a simulated social media environment in which people take on the role of a fake news creator and learn about six common misinformation techniques over the course of six levels, or "badges" (for a detailed theoretical overview see Roozenbeek and van der Linden, 2019; van der Linden and Roozenbeek, 2020). The inoculation component in the game consists of a combination of (a) warnings about fake news and (b) pre-exposure to weakened doses of the techniques used in the production of fake news. Both processes can potentially increase the inoculation effect by facilitating retention in memory for longer periods of time (Pfau et al., 1997, 2005). Research has shown that Bad News significantly improves players' ability to resist misinformation techniques after gameplay, and increase players' confidence in

\footnotetext{
${ }^{1}$ www.getbadnews.com
} 

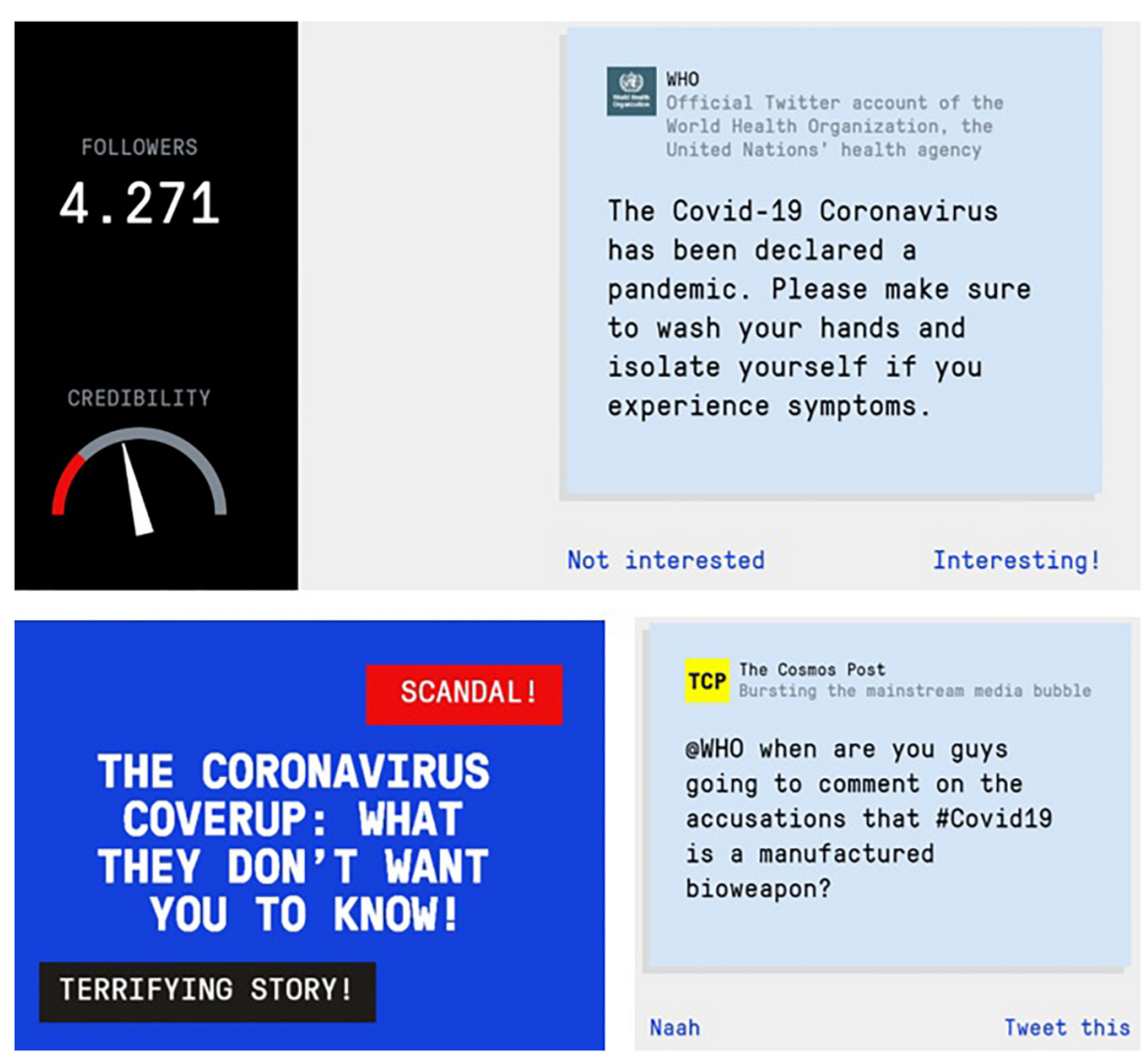

FIGURE 1 | Screenshots from the Bad News game about coronavirus (www.getbadnews.com). Images and links reproduced with permission from Bad News.

spotting misleading information (Basol et al., 2020). In addition, in collaboration with the United Kingdom Foreign Office, the game has been translated internationally and its effectiveness as an inoculation intervention has been replicated across five different language versions (Roozenbeek et al., 2020c). The inoculation effect itself can last for months (Pfau and Bockern, 1994), including with regular "top-ups" or "booster shots" following gameplay (Maertens et al., 2020b). In response to the outbreak of the coronavirus pandemic, we altered the Bad News game's "conspiracy" scenario to feature weakened doses of conspiracies about the virus. Figure 1 shows a number of screenshots from the game. Players are tasked with inventing and spreading a fake conspiracy theory about COVID-19, and learn about the negative consequences of their actions in the form of replies by social media users in their network, thus exposing how misinformation is created, spread and shared.

The relatively easy adaptation of the Bad News game to immunize people against misinformation specifically about the COVID-19 pandemic highlights the potential to translate theoretical laboratory findings into scalable real-world inoculation interventions: the game is played by about a million people worldwide (Roozenbeek et al., 2020c), thus "inoculating" a large number of people who voluntarily navigate to the Bad News website. Importantly, it is not necessary for every single individual to receive the "vaccine": if enough people have developed antibodies against the techniques used to spread misinformation about COVID-19, in theory, societal herd immunity could be achieved.

\section{CONCLUSION}

Prevention is better than cure. This is true as much for diseases as it is for the spread of misinformation. Although the Bad News game is a useful tool, more work is needed to curb the spread of misinformation about COVID-19, including a multi-layered defense system against "post-truth" science denial (van der Linden, 2019) which will include effective debunking and real-time rebuttal in addition to inoculation (Schmid and Betsch, 2019). A practical application of inoculation theory in the context of COVID-19 misinformation is the new online game, Go Viral!, ${ }^{2}$ developed in collaboration with the United Kingdom government and the WHO in which players learn to resist three manipulation techniques commonly used to spread misinformation about the coronavirus: fearmongering, the use of fake experts, and conspiracy theories. An open question in active inoculation research is the extent to which inoculation can boost truth-discernment skills, that is, not just the ability to spot and resist misinformation attacks but also the ability to better identify real or credible news (Guess et al., 2020; Roozenbeek et al., 2020a). Compton et al. (2016)

\footnotetext{
${ }^{2}$ The game can be played for free at www.goviralgame.com.
} 
called for more "work that pushes forward our understanding of persuasion and has applied value as a health messaging strategy to help combat serious threats to healthy living" (p. 1). Promoting accurate beliefs about COVID-19, and encouraging healthier, safer behaviors related to COVID-19 prevention, would certainly answer this call. Indeed, COVID-19 health messaging can harness both ways in which inoculation theory is used to protect healthier beliefs and actions: building resistance to unhealthy influence, like conspiracy theories, and encouraging healthier behaviors, like social distancing and wearing a mask in public. We look forward to future research on both prophylactic and therapeutic applications of psychological inoculation in the context of COVID-19.

\section{REFERENCES}

Ajzenman, N., Cavalcanti, T., and Da Mata, D. (2020). More than Words: Leaders' Speech and Risky Behavior during a Pandemic (No. 2034). Available online at: https://www.inet.econ.cam.ac.uk/working-paper-pdfs/wp2019.pdf (accessed July 25, 2020).

Allcott, H., Boxell, L., Conway, J. C., Gentzkow, M., Thaler, M., and Yang, D. Y. (2020). Polarization and Public Health: Partisan Differences in Social Distancing during the Coronavirus Pandemic (No. 26946). Available online at: https://www. nber.org/papers/w26946 (accessed October 13, 2020).

Andersen, K. G., Rambaut, A., Lipkin, W. I., Holmes, E. C., and Garry, R. F. (2020). The proximal origin of SARS-CoV-2. Nat. Med. 26, 450-452. doi: 10. 1038/s41591-020-0820-9

Banas, J. A., and Richards, A. S. (2017). Apprehension or motivation to defend attitudes? exploring the underlying threat mechanism in inoculation-induced resistance to persuasion. Commun. Monogr. 84, 164-178.

Basol, M., Roozenbeek, J., and van der Linden, S. (2020). Good news about bad news: gamified inoculation boosts confidence and cognitive immunity against fake news. J. Cogn. 3, 1-9. doi: 10.5334/joc.91

BBC News (2020). Ofcom: Covid-19 5G Theories are "Most Common" Misinformation. Www.Bbc.Co.Uk. Available online at: https://www.bbc.co.uk/ news/technology-52370616 (accessed April 21, 2020).

Bode, L., and Vraga, E. K. (2015). In related news, that was wrong: the correction of misinformation through related stories functionality in social media. J. Commun. 65, 619-638. doi: 10.1111/jcom.12166

Brady, W. J., Wills, J. A., Jost, J. T., Tucker, J. A., and Van Bavel, J. J. (2017). Emotion shapes the diffusion of moralized content in social networks. Proc. Natl. Acad. Sci. U.S.A. 114, 7313-7318. doi: 10.1073/pnas.1618923114

Carlsson, U. (2019). Understanding Media and Information Literacy MIL) in the Digital Age: A Question of Democracy. Gothenburg: University of Gothenburg.

Cohen, J. (2020). Scientists "Strongly Condemn" Rumors and Conspiracy theories about Origin of Coronavirus Outbreak. Science Magazine. Available online at: https://www.sciencemag.org/news/2020/02/scientists-strongly-condemnrumors-and-conspiracy-theories-about-origin-coronavirus

(accessed February 19, 2020).

Compton, J. (2013). "Inoculation theory," in The SAGE Handbook of Persuasion: Developments in Theory and Practice, 2nd Edn, eds J. P. Dillard and L. Shen (Thousand Oaks, CA: SAGE Publications), 220-236. doi: 10.4135/ 9781452218410

Compton, J. (2020). Prophylactic versus therapeutic inoculation treatments for resistance to influence. Commun. Theory 30, 330-343. doi: 10.1093/ct/qtz004

Compton, J., Jackson, B., and Dimmock, J. A. (2016). Persuading others to avoid persuasion: inoculation theory and resistant health attitudes. Front. Psychol. 7:122. doi: 10.3389/fpsyg.2016.00122

Constine, J. (2020). Facebook Deletes Brazil President's Coronavirus Misinfo Post. Tech Crunch. Available online at: https://techcrunch.com/2020/03/30/ facebook-removes-bolsonaro-video/ (accessed March 31, 2020).

Cook, J., Lewandowsky, S., and Ecker, U. K. H. (2017). Neutralizing misinformation through inoculation: exposing misleading argumentation

\section{DATA AVAILABILITY STATEMENT}

The original contributions presented in the study are included in the article/supplementary material, further inquiries can be directed to the corresponding author.

\section{AUTHOR CONTRIBUTIONS}

SL and JR conceptualized the study and drafted the manuscript. JC co-authored the article and provided input on the final version of the manuscript. All authors contributed to the article and approved the submitted version.

techniques reduces their influence. PLoS One 12:e0175799. doi: 10.1371/journal. pone.0175799

Cook, J., van der Linden, S., Lewandowsky, S., and Ecker, U. K. H. (2020). Coronavirus, "Plandemic" and the Seven Traits of Conspiratorial Thinking. The Conversation. Available online at: https://theconversation.com/coronavirusplandemic-and-the-seven-traits-of-conspiratorial-thinking-138483 (accessed May 15, 2020).

Cornwall, W. (2020). Officials gird for a war on vaccine misinformation. Science $369,14-15$.

Depoux, A., Martin, S., Karafillakis, E., Preet, R., Wilder-Smith, A., and Larson, H. (2020). The pandemic of social media panic travels faster than the COVID-19 outbreak. J. Travel Med. 27:taaa031.

Dimmock, J. A., Gagné, M., Proud, L., Howle, T. C., Rebar, A. L., and Jackson, B. (2016). An exercise in resistance: inoculation messaging as a strategy for protecting motivation during a monotonous and controlling exercise class. J. Sport Exerc. Psychol. 38, 567-578. doi: 10.1123/jsep.20160146

Dryhurst, S., Schneider, C. R., Kerr, J., Freeman, A. L., Recchia, G., Van Der Bles, A. M., et al. (2020). Risk perceptions of COVID-19 around the world. J. Risk Res. 1-13. doi: 10.1080/13669877.2020.1758193 [Epub ahead of print].

Ecker, U. K. H., Lewandowsky, S., and Tang, D. T. W. (2010). Explicit warnings reduce but do not eliminate the continued influence of misinformation. Mem. Cogn. 38, 1087-1100. doi: 10.3758/MC.38.8.1087

Freeman, D., Waite, F., Rosebrock, L., Petit, A., Causier, C., East, A., et al. (2020). Coronavirus conspiracy beliefs, mistrust, and compliance with government guidelines in England. Psychol. Med. 1-30. doi: 10.1017/S0033291720001890 [Epub ahead of print].

Geleris, J., Sun, Y., Platt, J., Zucker, J., Baldwin, M., Hripcsak, G., et al. (2020). Observational study of hydroxychloroquine in hospitalized patients with Covid-19. N. Engl. J. Med. 382, 2411-2418. doi: 10.1056/NEJMoa201 2410

Goga, O., Venkatadri, G., and Gummadi, K. P. (2015). “The Doppelgänger Bot Attack: exploring Identity Impersonation in Online Social Networks," in Proceedings of the 2015 Internet Measurement Conference, Tokyo, 141-153. doi: 10.1145/2815675.2815699

Guess, A. M., Lerner, M., Lyons, B., Montgomery, J. M., Nyhan, B., Reifler, J., et al. (2020). A digital media literacy intervention increases discernment between mainstream and false news in the United States and India. Proc. Natl. Acad. Sci. U.S.A. 117, 15536-15545.

Habersaat, K. B., Betsch, C., Danchin, M., Sunstein, C. R., Böhm, R., Falk, A., et al. (2020). Ten considerations for effectively managing the COVID-19 transition. Nat. Hum. Behav. 4, 677-687.

Imhoff, R., and Lamberty, P. (2020). A bioweapon or a hoax? The link between distinct conspiracy beliefs about the Coronavirus disease (COVID19) outbreak and pandemic behavior. Soc. Psychol. Person. Sci. doi: 10.1177/ 1948550620934692 [Epub ahead of print].

Jolley, D., and Douglas, K. M. (2017). Prevention is better than cure: addressing anti-vaccine conspiracy theories. J. Appl. Soc. Psychol. 47, 459-469. doi: 10.1111/ jasp. 12453 
Jolley, D., and Paterson, J. L. (2020). Pylons ablaze: examining the role of 5G COVID-19 conspiracy beliefs and support for violence. Br. J. Soc. Psychol. 59, 628-640.

Krause, N. M., Freiling, I., Beets, B., and Brossard, D. (2020). Fact-checking as risk communication: the multi-layered risk of misinformation in times of COVID-19. J. Risk Res. 1-8. doi: 10.1080/13669877.2020.1756385 [Epub ahead of print].

Lewandowsky, S., Ecker, U. K. H., Seifert, C. M., Schwarz, N., and Cook, J. (2012). Misinformation and its correction: continued influence and successful debiasing. Psychol. Sci. Public Interest 13, 106-131. doi: 10.1177/ 1529100612451018

Lewandowsky, S., Gignac, G. E., and Oberauer, K. (2013). The role of conspiracist ideation and worldviews in predicting rejection of science. PLoS One 8:e75637. doi: 10.1371/journal.pone.0075637

Li, H. O.-Y., Bailey, A., Huynh, D., and Chan, J. (2020). YouTube as a source of information on COVID-19: a pandemic of misinformation? BMJ Global Health 5:e002604. doi: 10.1136/bmjgh-2020-002604

Maertens, R., Anseel, F., and van der Linden, S. (2020a). Combatting climate change misinformation: evidence for longevity of inoculation and consensus messaging effects. J. Environ. Psychol. 70:101455.

Maertens, R., Roozenbeek, J., Basol, M., and van der Linden, S. (2020b). Longterm effectiveness of inoculation against misinformation: three longitudinal experiments. J. Exp. Psychol. Applied doi: 10.1037/xap0000315

McGuire, W. J. (1964). Inducing resistance against persuasion: some contemporary approaches. Adv. Exp. Soc. Psychol. 1, 191-229. doi: 10.1016/S0065-2601(08) 60052-0

McGuire, W. J., and Papageorgis, D. (1961). Resistance to persuasion conferred by active and passive prior refutation of the same and alternative counterarguments. J. Abnormal Soc. Psychol. 63, 326-332.

Meyerowitz, E. A., Vannier, A. G. L., Friesen, M. G. N., Schoenfeld, S., Gelfand, J. A., Callahan, M. V., et al. (2020). Rethinking the role of hydroxychloroquine in the treatment of COVID-19. FASEB J. 34, 6027-6037. doi: 10.1096/fj.20200 0919

Miller, C. H., Ivanov, B., Sims, J., Compton, J., Harrison, K. J., Parker, K. A., et al. (2013). Boosting the potency of resistance: combining the motivational forces of inoculation and psychological reactance. Hum. Commun. Res. 39, 127-155. doi: $10.1111 / j .1468-2958.2012 .01438 . x$

Mitchell, A., and Oliphant, J. B. (2020). Americans Immersed in COVID-19 News; Most Think Media Are Doing Fairly Well Covering It. Pew Research Center. Available online at: https://www.journalism.org/2020/03/18/americansimmersed-in-covid-19-news-most-think-media-are-doing-fairly-wellcovering-it/ (accessed March 18, 2020).

Ofcom (2020). Half of UK Adults Exposed to False Claims about Coronavirus. Available online at: https://www.ofcom.org.uk/about-ofcom/latest/featuresand-news/half-of-uk-adults-exposed-to-false-claims-about-coronavirus (accessed May 18, 2020).

Parker, K. A., Ivanov, B., and Compton, J. A. (2010). Inoculation's efficacy with young adults' risky behaviors: can inoculation confer cross-protection over related but untreated issues? Health Commun. 27, 223-233. doi: 10.1080/ 10410236.2011.575541

Pennycook, G., Cannon, T., and Rand, D. G. (2018). Prior exposure increases perceived accuracy of fake news. J. Exp. Psychol. 147, 1865-1880. doi: 10.1037/ xge 0000465

Pfau, M., and Bockern, S. V. (1994). The persistence of inoculation in conferring resistance to smoking initiation among adolescents: the second year. Hum. Commun. Res. 20, 413-430. doi: 10.1111/j.1468-2958.1994.tb0 0329.x

Pfau, M., Compton, J., Parker, K. A., An, C., Wittenberg, E. M., Ferguson, M., et al. (2006). The conundrum of the timing of counterarguing effects in resistance: strategies to boost the persistence of counterarguing output. Commun. Q. 54, 143-156. doi: 10.1080/01463370600650845

Pfau, M., Ivanov, B., Houston, B., Haigh, M., Sims, J., Gilchrist, E., et al. (2005). Inoculation and mental processing: the instrumental role of associative networks in the process of resistance to counterattitudinal influence. Commun. Monogr. 72, 414-441. doi: 10.1080/03637750500322578

Pfau, M., Roskos-Ewoldsen, D., Wood, M., Yin, S., Cho, J., Lu, K.-H., et al. (2003). Attitude accessibility as an alternative explanation for how inoculation confers resistance. Commun. Monogr. 70, 39-51. doi: 10.1080/715114663
Pfau, M., Tusing, J., Koerner, A. F., Lee, W., Godbold, L. C., Penaloza, L. J., et al. (1997). Enriching the inoculation construct the role of critical components in the process of resistance. Hum. Commun. Res. 24, 187-215. doi: 10.1111/j.14682958.1997.tb00413.x

Pfau, M., Van Bockern, S., and Kang, J. G. (1992). Use of inoculation to promote resistance to smoking initiation among adolescents. Commun. Monogr. 59, 213-230. doi: 10.1080/03637759209376266

Richards, A. S., and Banas, J. A. (2015). Inoculating against reactance to persuasive health messages. Health Commun. 30, 451-460.

Roozenbeek, J., Maertens, R., McClanahan, W., and van der Linden, S. (2020a). Differentiating item and testing effects in inoculation research on online misinformation. Educ. Psychol. Meas. 1-23. doi: 10.1177/0013164420940378 [Epub ahead of print].

Roozenbeek, J., Schneider, C. R., Dryhurst, S., Kerr, J., Freeman, A. L. J., van der Bles, A. M., et al. (2020b). Susceptibility to misinformation about COVID-19 around the world. R. Soc. Open Sci. 7:201199. doi: 10.1098/rsos.20 1199

Roozenbeek, J., and van der Linden, S. (2018). The fake news game: actively inoculating against the risk of misinformation. J. Risk Res. 22, 570-580. doi: $10.1080 / 13669877.2018 .1443491$

Roozenbeek, J., and van der Linden, S. (2019). Fake news game confers psychological resistance against online misinformation. Palgrave Commun. 5:65. doi: 10.1057/s41599-019-0279-9

Roozenbeek, J., van der Linden, S., and Nygren, T. (2020c). Prebunking interventions based on "inoculation" theory can reduce susceptibility to misinformation across cultures. Harvard Kennedy School (HKS) Misinform. Rev. 1, 1-15. doi: $10.37016 / / \mathrm{mr}-2020-008$

Roser, M., Ritchie, H., Ortiz-Ospina, E., and Hasell, J. (2020). Coronavirus Pandemic (COVID-19). Available online at: https://ourworldindata.org/ coronavirus (accessed May 18, 2020).

Sanders, L. (2020). The Difference between What Republicans and Democrats Believe to be True about COVID-19. YouGov. Available online at: https://today.yougov.com/topics/politics/articles-reports/2020/05/26/ republicans-democrats-misinformation (accessed May 26, 2020).

Schmid, P., and Betsch, C. (2019). Effective strategies for rebutting science denialism in public discussions. Nat. Hum. Behav. 3, 931-939. doi: 10.1038/ s41562-019-0632-4

Scott, M. (2020). Facebook's Private Groups are Abuzz with Coronavirus Fake News. Politico. Available online at: https://www.politico.eu/article/facebookmisinformation-fake-news-coronavirus-covid19/ (accessed March 30, 2020).

Spring, M. (2020). Coronavirus: The Human Cost of Virus Misinformation. BBC News. Available online at: https://www.bbc.com/news/stories-52731624 (accessed May 27, 2020).

Uscinski, J. E., Enders, A. M., KLofstad, C., Seelig, M., Funchion, J., Everett, C., et al. (2020). Why do people believe COVID-19 conspiracy theories? Harvard Kennedy School (HKS) Misinform. Rev. 1, 1-12. doi: 10.37016/mr-2020-015

Van Bavel, J. J., Baicker, K., Boggio, P. S., Capraro, V., Cichocka, A., Cikara, M., et al. (2020). Using social and behavioural science to support COVID-19 pandemic response. Nat. Hum. Behav. 4, 460-471. doi: 10.1038/s41562-020-0884-z

van der Linden, S. (2015). The conspiracy-effect: exposure to conspiracy theories (about Global Warming) decreases pro-social behavior and science acceptance. Pers. Individ. Diff. 87, 171-173.

van der Linden, S. (2019). Countering science denial. Nat. Hum. Behav. 3, 889-890. doi: 10.1038/s41562-019-0631-5

van der Linden, S., Leiserowitz, A., Rosenthal, S., and Maibach, E. (2017). Inoculating the public against misinformation about climate change. Global Challenges 1:1600008. doi: 10.1002/gch2.201600008

van der Linden, S., and Roozenbeek, J. (2020). "Psychological inoculation against fake news," in The Psychology of Fake News: Accepting, Sharing, and Correcting Misinformation, eds R. Greifenader, M. Jaffé, E. Newman, and N. Schwarz (London: Psychology Press).

Vosoughi, S., Roy, D., and Aral, S. (2018). The spread of true and false news online. Science 359, 1146-1151. doi: 10.1126/science.aap9559

Wong, N. C., and Harrison, K. J. (2014). Nuances in Inoculation: protecting positive attitudes towards the HPV vaccine and the practice of vaccinating children. J. Women's Health Issues Care 3:6. doi: 10.4172/2325-9795.100 0170 
World Health Organization (2020a). Coronavirus disease (COVID-19) advice for the public: Mythbusters. Available online at: https:/www.who.int/emergencies/ diseases/novel-coronavirus-2019/advice-for-public/myth-busters (accessed May 5, 2020).

World Health Organization (2020b). Novel Coronavirus (2019-nCoV) Situation Report - 13. Available online at: https://www.who.int/docs/default-source/ coronaviruse/situation-reports/20200202-sitrep-13-ncov-v3.pdf (accessed February 2, 2020).

Zarocostas, J. (2020). How to fight an infodemic. Lancet 395:676. doi: 10.1016/ S0140-6736(20)30461-X
Conflict of Interest: The authors declare that the research was conducted in the absence of any commercial or financial relationships that could be construed as a potential conflict of interest.

Copyright (c) 2020 van der Linden, Roozenbeek and Compton. This is an open-access article distributed under the terms of the Creative Commons Attribution License (CC BY). The use, distribution or reproduction in other forums is permitted, provided the original author(s) and the copyright owner(s) are credited and that the original publication in this journal is cited, in accordance with accepted academic practice. No use, distribution or reproduction is permitted which does not comply with these terms. 